\title{
Variables demográficas y engrandecimiento marital en Monterrey, México
}

José Moral de la Rubia*

Doctor en Filosofía y Ciencias de la Educación, Universidad de Alcalá de Henares, España. Profesor investigador, Facultad de Psicología, Universidad Autónoma de Nuevo León, México. Correo electrónico:

jose_moral@hotmail.com

Recibido: 9 de marzo del 2016 Aprobado: 22 de junio del 2016

Cómo citar este artículo: Moral de la Rubia, J. (2016). Variables demográficas y engrandecimiento marital en Monterrey, México. Pensando Psicología, 12(20), 13-28. doi: http://dx.doi.org/10.16925/ pe.v12i20.1560

\section{Resumen}

Introducción: el engrandecimiento marital es una tendencia a idealizar a la pareja. Se considera un rasgo funcional correlacionado con la satisfacción con la relación. Existe una escala para su evaluación creada en Canadá (18 ítems) y adaptada en México (14 ítems), pero no está baremada. Objetivos: 1) describir la distribución de la Escala de Engrandecimiento Marital en su formato original de 18 ítems dicotomizados (EEM) y en su versión de 14 ítems ordinales (EEM14), a fin de comprobar cuál se adapta mejor a un concepto de rasgo funcional; y 2) estudiar la relación de la EEM y la EEM14 con variables sociodemográficas y de religiosidad (convicción y práctica), a fin de comprobar la sustantividad de estos correlatos al controlar el efecto de la satisfacción con la relación. Metodología: se aplicó la EEM, la Escala de Valoración de la Relación (RAs) y un cuestionario de datos sociodemográficos a 431 mujeres y 376 hombres casados o en unión libre de Monterrey, México. Se usó un muestreo probabilístico de rutas aleatorias. Resultados: la EEM mostró desviación de la normalidad con asimetría positiva y perfil aplanado. La EEM14 mostró ligera desviación de la normalidad con ligera asimetría negativa y perfil mesocúrtico. La EEM y la EEM14 fueron independientes o presentaron asociaciones bajas con las variables sociodemográficas y de religiosidad. Solo las correlaciones con religiosidad fueron significativas y no triviales tras parcializar el efecto de la satisfacción con la relación (RAS). Conclusiones: la distribución de la EEM14 es más congruente con el concepto de rasgo funcional que pretende medir y no requiere baremos diferenciales por variables sociodemográficas.

Palabras clave: deseabilidad social, engrandecimiento marital, psicometría, relación de pareja, religiosidad. 


\title{
Demographic Variables and Marital Aggrandizement in Monterrey, Mexico
}

\begin{abstract}
Introduction: Marital aggrandizement is a tendency to idealize one's partner. It is considered to be a functional trait correlated to satisfaction with the relationship. There is a scale for its assessment created in Canada (18 items) and adapted in Mexico (14 items), but is not validated. Objectives: 1) To describe the distribution of the Marital Aggrandizement Scale in its original format of 18 dichotomized items (MAS) and its version of 14 ordinal items (MAS14), checking which is better suited to the concept of functional trait; and 2) to study the relationship of MAS and MAs14 with sociodemographic and religiosity (belief and practice) variables, verifying the substantiation of these correlates in controlling the effect of satisfaction with the relationship. Methodology: MAS, Relationship Assessment Ratio (RAS), and a sociodemographic data questionnaire were applied to 431 women and 376 men married or cohabiting in Monterrey, Mexico. Probability sampling of random routes was used. Results: MAS show deviation from normality with positive asymmetry and flattened profile. MAs14 showed slight deviation from normality with slight negative asymmetry and mesokurtic profile. MAS and MAs14 were independent or exhibited low associations with sociodemographich and religiosity variables. Only correlations with religiosity were significant and nontrivial after biasing the effect of satisfaction with the relationship (RAS). Conclusions: The distribution of MAs14 is more consistent with the concept of functional feature it intends to measure and does not require differential scales for sociodemographic variables.
\end{abstract}

Keywords: social desirability, marital aggrandizement, psychometry, relationship, religiosity.

\section{Variáveis demográficas e engrandecimento conjugal em Monterrey, México}

\section{Resumo}

Introdução: o engrandecimento conjugal é uma tendência de idealização do par. É considerado um traço funcional correlacionado com a satisfação com o relacionamento. Existe uma escala para sua avaliação que foi criada no Canadá (18 itens) e depois adaptada no México (14 itens), mas ainda não foi escalada. Objetivos: 1) descrever a distribuição da Escala de Engrandecimento Conjugal no seu formato original de 18 itens dicotomizados (EEM, por suas siglas em espanhol) e na sua versão de 14 itens ordinais (EEM14), verificando qual se adapta melhor a um conceito de traço funcional; e 2) analisar a relação da EEM e EEM14 com variáveis sócio-demográficas e de religiosidade (convicção e pratica), verificando a sustantividade de tais correlatos no controle do efeito da satisfação com o relacionamento. Metodologia: aplicou-se a EEM, a Escala de Avaliação do Relacionamento (EAR) e um questionário de dados sócio-demográficas em 431 mulheres e 376 homens casados ou em união livre da cidade de Monterrey, México. Foi utilizada uma amostragem probabilística de rotas randômicas. Resultados: a EEM mostrou desviacão da normalidade com assimetria positiva e curva achatada. A EEM14 mostrou uma leve desviacão da normalidade com leve assimetria negativa e curva mesocúrtica. A EEM e EEM14 foram independentes ou apresentaram associações baixas com as variáveis sócio-demográficas e de religiosidade. Apenas as correlações com religiosidade foram significativas e não triviais após parcializar o efeito da satisfação com o relacionamento (RAS). Conclusões: a distribuição da EEM14 é mais congruente com o conceito de traço funcional que pretende medir e não requer escalas diferenciais por variáveis sócio-demográficas.

Palavras chave: desejabilidade social, engrandecimento conjugal, psicometria, relacionamento de casal, religiosidade. 


\section{Introducción}

En 1967, Edmonds desarrolló la Escala de Convencionalismo Marital (EEM), a fin de controlar el sesgo a dar respuestas socialmente deseables al momento de reportar satisfacción con la relación marital. No obstante, las correlaciones de la EEM con otras escalas de deseabilidad social fueron bajas $(r<0,30)$, cuando sus correlaciones con escalas de satisfacción con la relación y ajuste diádico fueron muy altas $(\mathrm{r} \geq 0,70)$, por lo cual se ha considerado que la EEM evalúa una distorsión idealista de la satisfacción con la relación (Fowers y Appeglate, 1996).

O’Rourke et al. (1996) acuñaron el término de engrandecimiento marital para denominar el rasgo evaluado por la EEM. El engrandecimiento marital se define como un estilo de respuesta que conduce a reportar una imagen exageradamente positiva del cónyuge y la relación marital. El engrandecimiento marital es un sesgo sistemático que se pone de manifiesto al evaluar la propia relación de pareja sin implicar necesariamente distorsiones idealistas en otros contextos. El engrandecimiento marital se concibe como unidimensional, esto es, todas las manifestaciones de engrandecimiento del cónyuge y la relación marital son indicadores de un rasgo unitario, con lo que no se puede distinguir entre procesos deliberados de engaño (manejo de la impresión) y procesos implícitos de distorsión (autoengaño), tal como postula el modelo bidimensional de la deseabilidad social. Además, se define como un rasgo funcional que no implica necesariamente psicopatología (O’Rourke y Cappeliez, 2002).

Aunque el ajuste diádico, la satisfacción y la durabilidad de la relación no requieren que el sesgo del engrandecimiento marital esté destacado, se observa que la presencia del mismo los facilita (Moral, 2008a; O’Rourke y Cappeliez, 2003a). Se considera que el engrandecimiento forma parte del enamoramiento o amor hacia la pareja (Gagné y Lydon, 2004; Swami et al., 2009), ya sea que la pareja se encuentre en una fase inicial de amor pasional, o en una fase de compromiso amoroso (Díaz-Loving y Rivera-Aragón, 2010).

El efecto positivo del engrandecimiento en la calidad de la relación se observa claramente en situaciones de precariedad, crisis y dificultades para la supervivencia de la relación, como por ejemplo una enfermedad crónica en el cónyuge (O'Rourke et al., 2011; O’Rourke, Gomes y Davidov, 2012; O’Rourke y Tuokko, 2000; O’Rourke y Wenaus, 2010). En estos casos la presencia de un mayor engrandecimiento marital se atribuye al mecanismo cognitivo de la reducción de la disonancia (O’Rourke et al., 2011; O’Rourke y Cappeliez, 2003a).

Para la evaluación del engrandecimiento marital y como alternativa a la escala de Edmonds (1967), O’Rourke (2001) creó la Escala de Engrandecimiento Marital (EEM) de 18 ítems tipo Likert, con siete opciones de respuesta. O’Rourke y Cappeliez (2002) reportaron que, en conformidad con las expectativas de constructo, la estructura factorial de la EEM se ajustó bien a un modelo unidimensional, y que la EEM compartió el 28,1\% de su varianza con la Escala de Ajuste Diádico de Spanier (1976), el 22,1\% con el factor de autoengaño, y el 11,6\% con el factor de manejo de la impresión del Inventario Balanceado de Respuestas Socialmente Deseables de Paulhus (1998). Además, los autores indicaron que la EEM fue independiente de la edad, la afiliación religiosa, la frecuencia de asistencia a los servicios religiosos, la escolaridad, los años de matrimonio y el número de matrimonios (O’Rourke y Cappeliez, 2002). Asimismo, las medias de hombres y mujeres en la EEM fueron estadísticamente equivalentes, y el modelo de medida de un factor presentó buenas propiedades de invarianza por sexo (O’Rourke y Cappeliez, 2001).

La EEM se puntúa asignando uno a las dos opciones extremas y cero a las cinco opciones restantes. Al sumar los ítems se obtiene una puntuación con un rango potencial de 0 a 18. En una muestra no probabilística de 532 adultos mayores, O’Rourke y Cappeliez (2001) reportaron una media de 5,53 (IC 95\%: 5,11, 5,94; $\mathrm{DE}=4,24)$. En otra muestra no probabilística de 198 adultos mayores, O'Rourke (2002) reportó una media de 5,46 (IC 95\%: 4,83, 6,09; DE = $3,95)$; en una segunda aplicación realizada 16 semanas después, obtuvo una media de 5,18 (IC 95\%: 4,67, 5,69; $\mathrm{DE}=3,19)$, siendo ambas medias estadísticamente equivalentes $(t[196]=1,52, p>0,05)$ y la correlación entre ambas puntuaciones muy alta (r[198] $=0,79$ ). Estos datos muestran que la escala posee estabilidad, en conformidad con el constructo de rasgo o la tendencia de la personalidad.

La EEM en su versión original se redactó en inglés, pero ha sido traducida por los autores al francés, usando el método de traducción inversa (O’Rourke y Cappeliez, 2003b). En una muestra de 61 participantes francófonos canadienses, la media de la versión francesa fue 5,84 (IC 95\%: 5,66, 6,03, DE = 4,99), la cual fue una media estadísticamente equivalente 
a la media que O’Rourke y Cappeliez (2001) habían obtenido previamente en una muestra de 532 adultos mayores anglófonos ( $\mathrm{M}=5,53$, IC 95\%: 5,11, 5,94). De nuevo, la escala fue independiente de la edad, la escolaridad, el estatus socioeconómico, la afiliación religiosa, la frecuencia de asistencia a los servicios religiosos y los años de matrimonio (O’Rourke y Cappeliez, 2003b).

La EEM ha sido traducida al hebreo por traducción inversa (O’Rourkeet al., 2012). Se aplicó la versión de la EEM en hebreo a una muestra de 206 adultos mayores israelíes, y se aplicó la versión en inglés a otra muestra de 215 adultos mayores canadienses. La media de los 206 participantes israelíes fue 5,48 (IC 95\%: $4,85,6,11, \mathrm{DE}=4,02)$ y la de los 215 canadienses fue 4,86 (IC 95\%: 4,26, 5,46, DE =3,90), sin diferencia estadísticamente significativa entre ambas medias ( $\mathrm{t}[413]$ $=1,61, \mathrm{p}>0,05)$. Además, el modelo de un factor mostró propiedades aceptables de invarianza entre las muestras de ambos países.

La EEM también ha sido traducida al español por traducción inversa (Moral, 2007). En una muestra no probabilística de 100 parejas mexicanas de población general, Moral (2007) obtuvo una media de 5,87 (IC 95\%: 5,32, 6,42; $\mathrm{DE}=2,95)$. Esta media fue estadísticamente equivalente a la reportada por O'Rourke y Cappeliez (2001) en una muestra de 532 adultos mayores con la versión de la escala en inglés ( $\mathrm{t}[199]=$ $1,22, p=0,225)$; a la media reportada por O'Rourke y Cappeliez (2003b) en una muestra 61 adultos mayores con la versión en francés $(\mathrm{t}[199]=0,11, \mathrm{p}=0,915)$; $\mathrm{y}$ a la reportada por O'Rourke et al. (2012) en una muestra de 206 adultos mayores israelíes $(\mathrm{t}[199]=1,40, \mathrm{p}=$ $0,164)$. Entre los 200 participantes mexicanos, la distribución de las puntuaciones en la EEM no se ajustó a una curva normal ( $\mathrm{zK}-\mathrm{s}=0,112, \mathrm{p}<0,001)$, sino que mostró un perfil asimétrico positivo y platicúrtico, esto es, un sesgo hacia la puntuaciones bajas y una distribución más homogénea de lo que corresponde a una curva normal. Esta homogeneidad en la distribución indica que el rasgo evaluado es usual. La distribución de un rasgo inusual mostraría un fuerte apuntamiento en los valores más bajos (Cohen et al., 2012; Fischer y Milfont, 2010).

$\mathrm{Al}$ momento de obtener las puntuaciones en la escala por medio de la suma simple de ítems, Moral (2007) sugirió usar los siete valores discretos asignados a las respuestas dadas a los ítems en lugar de dicotomizar estas respuestas. De tal modo, se tiene un rango más amplio de puntuaciones (de 18 a 126), lo cual facilita que su distribución se ajuste a una curva normal, que es el tipo de distribución característica de los rasgos adaptativos o funcionales y no psicopatológicos (Cohen et al., 2012; Fischer y Milfont, 2010), como se postula sobre el engrandecimiento marital (O'Rourke y Cappeliez, 2002). Desde los datos de Moral (2007), la media de la ЕEм fue 76,52 (IC 95\%: $74,38,78,67 ; \mathrm{DE}=15,37$ ), con lo que se ajusta la distribución de sus puntuaciones a una curva normal (zK$\mathrm{s}=0,72, \mathrm{p}=0,681$ ).

Si se divide la puntuación-suma por el número de ítems sumados, se facilita la comparación de distintas versiones de la escala que difieran en el número de ítems y se posibilita la interpretación de las puntuaciones. Se facilita la comparación, ya que este cociente proporciona un rango continuo de valores entre un valor mínimo de 1 y un valor máximo de 7 , con independencia del número de ítems sumados. Se posibilita la interpretación, ya que el rango continuo se puede dividir en siete intervalos de amplitud constante ([Máx-Mín]/número de intervalos = [7 $-1] / 7=0,857)$, en correspondencia con los siete valores discretos asignados a las respuestas a cada ítem. Desde esta correspondencia, una puntuación entre 1 y 1,857 se interpreta como una respuesta de total desacuerdo, con una evaluación exageradamente positiva de la relación de pareja; entre 1,858 y 2,714 como una respuesta de bastante desacuerdo; entre 2,715 y 3,571 como una respuesta de ligero desacuerdo; entre 3,572 y 4,429 como una respuesta neutra; entre 4,430 y 5,286 como una respuesta de ligero acuerdo; entre 5,287 y 6,143 como una respuesta de bastante acuerdo; y entre 6,144 y 7 como una respuesta de total acuerdo. Desde los datos de Moral (2007), la media de la Еем fue 4,25 (IC 95\%: 4,13, 4,37; DE =0,85) y correspondió a la opción de respuesta neutra "ni de acuerdo ni en desacuerdo".

En otro estudio realizado en México, usando una muestra probabilística de 807 personas casadas o en unión libre, Moral (s. f. a) aconsejó eliminar cuatro ítems (el ítem directo $7 \mathrm{y}$ los ítems inversos 11,13 y 17) para incrementar la consistencia interna de la escala ( $\alpha$ ordinal $=0,87$ con 14 ítems [Eem14] versus 0,85 con 18 ítems [EEM]), y mejorar el ajuste de un modelo de un factor con todos sus residuos de medida independientes. Por el método de mínimos cuadrados libres de escala, el ajuste a los datos del modelo de un factor con 14 indicadores y todos sus residuos de medida independientes varió de bueno (GFI $=0,98$, AGFI $=0,97, \mathrm{NFI}=0,96$ y RFI $=0,95)$, a aceptable $(\chi 2 / \mathrm{gl}=$ 4,69 y SRMR $=0,07$ ), y su bondad de ajuste fue significativamente mejor que la del modelo de un factor con 
18 indicadores $(\Delta \chi 2[58]=613,62, \mathrm{p}<0,001)$. Moral (s. f. b) reportó que la media de los hombres en la escala fue estadísticamente equivalente a la de las mujeres, y el modelo de un factor con 14 indicadores y todos sus residuos de medida independientes tuvo unas propiedades de invarianza por sexo aceptables $(\chi 2 / \mathrm{gl}=2,77, \mathrm{GFI}=0,97, \mathrm{AGFI}=0,96, \mathrm{NFI}=0,95, \mathrm{RFI}$ $=0,95$ y SRMR $=0,07$ en el modelo sin restricciones), aunque el ítem 9 sobre perfecta comprensión mutua y el ítem 18 sobre la perfecta elección de pareja presentaron unas cargas factoriales significativamente mayores en mujeres que en hombres.

Desde los resultados de estudios realizados con muestras no probabilísticas, se colige que la EEM puntuada con un rango de 0 a 18 presenta asimetría positiva o sesgo hacia los valores bajos, así como una distribución amplia y más homogénea que la que corresponde con una curva normal. Esta distribución refleja un rasgo usual, pero no claramente convencional, al presentar una expresividad muy variable. Si la EEM se puntúa con un rango continuo de 1 a 7 , su distribución es normal, como las escalas de satisfacción con la relación, con las cuales presenta una correlación alta (Moral, 2007). Con este mayor rango reflejaría un rasgo convencional que facilita o predice un buen ajuste diádico (Moral, 2008a) y satisfacción con la relación (Moral, s. f. a), siendo el engrandecimiento marital claramente discernible de ajuste diádico y satisfacción con la relación.

Cabe preguntarse cuál sería la conceptualización y la forma de baremar más adecuadas para la escala en la población general de Monterrey, México. Si la distribución de las puntuaciones se ajustara a una curva normal - lo cual es propio de un rasgo expresivo sometido a reglas de convencionalismo o adaptación social—, se debería baremar por la media y la desviación estándar. Si la distribución mostrara sesgo positivo y aplanamiento - lo cual es propio de un rasgo no convencional-, se debería baremar por los cuantiles. Si la distribución fuera asimétrica positiva y apuntada - lo cual es propio de un rasgo desadaptativo-, también se debería baremar por los cuantiles (Cohen et al., 2012; Fischer y Milfont, 2010). En muestras no probabilísticas, la EEM es independiente no solo del sexo, sino también de otras variables, tales como la edad, la escolaridad, el estatus socioeconómico, la religiosidad y los años de matrimonio. Cabe preguntarse si estos hallazgos se sostienen en una muestra probabilística, y si la EEM es también independiente del estatus marital (casado o unión libre). Debe señalarse que las parejas casadas reportan más satisfacción con la relación y ajuste diádico, y sus relaciones son más persistentes que las parejas que cohabitan (Boyes y Fletcher, 2007). A su vez, por la correlación alta entre la EEM y la satisfacción con la relación, sería importante contrastar la sustantividad de cualquier correlato sociodemográfico de la EEM una vez controlado el efecto de la satisfacción con la relación, ya que podría tratarse de un correlato espurio explicado por la correlación de la variable sociodemográfica con la satisfacción con la relación (FanniAsl et al., 2009; Jose y Alfons, 2007).

La satisfacción con la relación no está claramente relacionada con el sexo. Un estudio de metaanálisis reveló que la menor satisfacción con la relación observada en las mujeres era atribuible a las muestras clínicas (Jackson et al., 2014). No obstante, en población general, menor satisfacción con la relación sí se ha relacionado con mayor número de hijos (Carlson et al., 2014; Wendorf et al., 2011); mayor edad (Dellner, 2008; Britt et al., 2008); más años de relación (Dellner, 2008); menor escolaridad (Britt et al., 2008); menores ingresos económicos (Carlson et al., 2014; Glenwright y Fowler, 2013); mayor religiosidad (Ahmadi et al., 2008; Glenwright y Fowler, 2013); y menor estatus laboral (Bodenmann et al., 2007). Todas estas correlaciones suelen ser bajas $(<0,30)$ y se destaca la del número de hijos (Moral, 2015).

En la presente investigación, realizada con una muestra probabilística de población de parejas casadas y en unión libre de la ciudad de Monterrey en México, se va a considerar el modelo unidimensional de 18 indicadores con su rango discreto de puntuaciones de 0 a 18 (EEM), originariamente formulado por O'Rourke y Cappeliez $(2001,2002)$. De igual forma, el de 14 indicadores con su rango continuo de puntuaciones de 1 a 7 (ЕЕм14), propuesto por Moral (s. f. a). Retomando las preguntas formuladas, este estudio tiene como objetivos: 1) Describir la distribución de la EEM y la EEM14, comprobando cuál se adapta mejor a un concepto de rasgo funcional; 2) Estudiar la relación de ambas versiones con 10 variables sociodemográficas (estatus marital, afiliación religiosa, actividad u ocupación laboral, edad, escolaridad, ingresos económicos, número de hijos que se tiene con la pareja actual, número total de hijos que se tiene, número de parejas previas con las que se ha estado casado o en unión libre y tiempo de relación con la presente pareja), y dos variables de religiosidad (convicción religiosa y frecuencia de asistencia a los 
servicios religiosos); y 3) Comprobar la significación de cualquier correlación una vez parcializado el efecto de la satisfacción con la relación.

En relación con los objetivos formulados, se espera que la distribución de las puntuaciones en EEM presente asimetría positiva y aplanamiento, y que en EEM14 se ajuste a un modelo de curva normal (O’Rourke, 2002; Moral, 2007); así mismo, que tanto la EEM como la EEM 14 sean independientes o tengan unas correlaciones bajas con las variables sociodemográficas y de religiosidad (O’Rourke y Cappeliez, 2002; O'Rourke y Cappeliez, 2003b; y que cualquier correlación significativa finalmente sea espuria una vez parcializado el efecto de la satisfacción con la relación (Fanni-Asl et al., 2009; Jose y Alfons, 2007).

\section{Método}

Se realizó un estudio no experimental con un diseño ex post facto transversal. Dado su primer objetivo, también constituye un estudio instrumental al centrarse en las propiedades distribucionales de un instrumento de medida (Sampieri et al., 2010).

\section{Participantes}

Los criterios de inclusión fueron: tener al menos 18 años, estar casado o vivir en unión libre con una pareja del sexo opuesto, residir en Monterrey, saber leer y escribir, y prestar el consentimiento informado. Los criterios de eliminación fueron: cuestionario incompleto e informar que la pareja estuvo presente al momento de diligenciarlo.

Se empleó un muestreo probabilístico usando el método de las rutas aleatorias, a fin de cubrir el primer objetivo de baremar la escala. De forma aleatoria se seleccionaron 80 direcciones de la ciudad a partir de la Guía Roji (dos por cada encuestador). Cada dirección se tomó como punto de partida de un itinerario. En cada itinerario se recolectaron 10 unidades. Se pretendía que fueran cinco mujeres y cinco hombres independientes, un participante por casa. El encuestador tocaba el timbre o la puerta de la casa. Si abrían la puerta, el entrevistador se presentaba como un ayudante de investigación, se informaba de los objetivos del estudio (estudio sobre dinámica de la vida marital o de convivencia por medio de un cuestionario), del responsable del mismo (autor del artículo), y criterios de inclusión. Si la persona deseaba participar voluntariamente y satisfacía los criterios de inclusión, se dejaba un cuestionario para recogerlo media hora después o cuando lo indicara la persona. Se instruía que debía contestarlo a solas, sin que su pareja leyera las preguntas o respuestas. Al recogerlo, se preguntaba si la pareja había estado presente a la hora de contestar y si había visto sus contestaciones. En caso de que no, se revisaba que estuviera todo contestado; si había preguntas vacías, se pedía amablemente que se respondiera. En caso de que sí, no se incluía en la base de datos. Los entrevistadores fueron alumnos de primer semestre de la facultad de psicología de la Universidad Autónoma de Nuevo León. La muestra fue reclutada de enero a mayo del 2014.

Con el fin de determinar el tamaño de la muestra se empleó el último censo de población y vivienda, en el cual se informa que la ciudad de Monterrey tiene 1135550 habitantes; la población adulta representa dos tercios de la pirámide poblacional, de modo que 750000 regiomontanos son adultos; entre los adultos, el 55,7\% están casados o viven en unión libre; el $34,6 \%$ son solteros; el $5 \%$ son viudos, el 2,8\% están separados y el 1,9\% están divorciados, con lo cual la población objeto de estudio se reduce a 417750 adultos (Instituto Nacional de Estadística, Geografía e Informática [INEGI], 2011a). Bajo un modelo de curva normal, si se desea estimar la media de la EEM con una desviación estándar esperada de 0,85 en un rango de 1 a 7 (Moral, 2007), y con un error absoluto de estimación de 0,059 ( $\mathrm{M}=4,25$, IC 95\%: 4,19,4,31), se requeriría una muestra de 807 individuos, esto es, una fracción de muestreo de $0,2 \%$. Así, se recolectó una muestra de 807 participantes voluntarios con todos sus datos completos.

De los 807 participantes, 431 (53,4\%) fueron mujeres y 376 (46,6\%) fueron hombres, sin diferencia de frecuencia estadísticamente significativa por la prueba binomial $(p=0,057)$. Con respecto al estado civil, 727 (90\%) de los 807 participantes indicaron estar casados y 80 (10\%) señalaron cohabitar en unión libre. La media de años de vivir juntos fue 11,61 (DE = 9,33), variando de seis meses a 43 años. De las 807 personas encuestadas, 677 (84\%) dijeron tener hijos y $130(16 \%)$ dijeron no tenerlos. La media de hijos totales fue $1,99(\mathrm{DE}=1,30)$, variando de 0 a 9. La media de edad fue de 35,40 años $(\mathrm{DE}=10,41)$ y la mediana fue de 34 años, variando de 18 a 94 años. La mediana de escolaridad $(\mathrm{Mdn}=2)$ correspondió a secundaria (de 7 a 9 años de escolaridad), y la media $(\mathrm{M}=2,54)$ quedó entre estudio de secundaria y media superior (en torno a 10 años de escolaridad). Con respecto a la 
actividad u ocupación laboral, 201 de los 807 participantes $(24,9 \%)$, indicaron dedicarse a su hogar; 237 $(29,4 \%)$ estar contratados como trabajadores manuales; $126(15,6 \%)$, estar contratados como trabajadores o empleados técnicos; 102 (12,6\%), estar contratados como empleados profesionales; 58 (7,2\%), tener empresa propia de pequeño tamaño o familiar; 42 $(5,2 \%)$, ser estudiantes; 23 (2,9\%), estar desempleados; y 18 (2,2\%), estar jubilados. La mediana y la media de ingreso mensual de la familia correspondieron al rango de 6000 a 11999 pesos mexicanos (de 400 a 800 dólares estadounidenses). Con respecto a la afiliación religiosa, 662 de los 807 participantes (82\%) dijeron ser cristianos católicos; 76 (9,4\%), ser cristianos no católicos; 13 (1,6\%), pertenecer a otras religiones, y 56 (6,9\%), no pertenecer a ninguna.

En la población de Monterrey se reportan 98 hombres por cada 100 mujeres. Una mediana de 30 años de edad que sube a 34 al eliminar el $31 \%$ de la población menor de 18 años; una media de 9,5 años de escolaridad (entre primer semestre y segundo de educación media superior); una media de ingresos mensuales de 6700 pesos mexicanos; una media de dos hijos; y el 83\% de la población con afiliación religiosa cristiana católica, el 7,4\%, cristiana no católica; el 2,5\%, de otra religión, y el 6,9\%, sin religión (INEGI, 2011a). Estos datos poblacionales son estadísticamente equivalentes a los de la muestra en la frecuencia de ambos sexos $(\chi 2[1, \mathrm{~N}=807]=1,872, \mathrm{p}=$ $0,171)$, y de la afiliación religiosa $(\chi 2[3, \mathrm{~N}=807]=$ $7,125, \mathrm{p}=0,068)$, así como en la media de hijos ( $\mathrm{t}[806]$ $=-0,270, p=0,787)$; a su vez, las medianas poblacionales de edad, escolaridad e ingresos son semejantes a las muestrales.

\section{Instrumentos}

Escala de Engrandecimiento Marital (O'Rourke, 2001) con la traducción al español de Moral (2007). La EEM evalúa la distorsión, en un sentido de exagerar las cualidades positivas y minimizar las negativas al momento de reportar la satisfacción con la relación. Consta de 18 ítems tipo Likert con un rango de respuestas de 1 ("totalmente en desacuerdo") a 7 ("totalmente de acuerdo"). Catorce de sus 18 ítems son directos al rasgo del engrandecimiento, y cuatro son inversos (ítems 2, 11, 13 y 17). Hay dos modos de puntuar la escala. Se asigna uno a los valores seis y siete, salvo en los cuatro ítems inversos en que esta asignación se hace a los valores uno y dos; y a los restantes valores se les asigna cero. $\mathrm{Al}$ sumar los unos se tiene la puntuación en la escala dentro de un rango de 0 a 18. Una puntuación mayor o igual que siete refleja una tendencia al engrandecimiento significativa (O’Rourke y Cappeliez, 2003b). Otra forma es sumar el valor numérico que corresponde a la respuesta dado a cada ítem ( 1 = "totalmente en desacuerdo", 2 = "bastante en desacuerdo", 3 = "algo en desacuerdo", $4=$ "ni de acuerdo ni en desacuerdo", 5 = "algo de acuerdo", 6 = "bastante de acuerdo" y 7 = "totalmente de acuerdo"), invirtiendo este valor en los ítems 2 , 11, 13 y 17 (8 - valor de respuesta); y dividir la suma por el número de ítems sumados, lo que proporciona puntuaciones en un rango continuo de 1 a 7 . Desde los datos de Moral (2007), una puntuación en la EEM $\geq 5,11$ muestra desviación de la media. O'Rourke y Cappeliez (2002) reportaron una consistencia interna buena, variando de 0,84 a 0,87 al ser calculada con el coeficiente a de Cronbach, y una estabilidad alta al ser estimada en un intervalo de 15 meses $(r[200]=$ $0,80)$. En la presente muestra, la consistencia interna de los 18 ítems fue buena ( $\alpha$ de Cronbach $=0,82$ y a ordinal = 0,85). Al eliminar los ítems 7, 11, 13 y 17, la consistencia interna también fue buena, pero ligeramente más alta ( $\alpha$ de Cronbach $=0,84$ y a ordinal $=$ 0,87) (Moral, s. f. a).

Escala de Valoración de la Relación (Relationship Assessment Scale [RAS]; Hendrick, 1988) con la validación en México por Moral (2008b). La Ras evalúa la satisfacción con la relación de pareja. La escala está integrada por siete ítems tipo Likert que tienen un rango de valores de uno a cinco. La puntuación se obtiene por suma simple de los ítems. Dos ítems están redactados en sentido contrario a la satisfacción (ítems 4 y 7). La escala presenta un recorrido potencial de 7 a 35. Una mayor puntuación refleja una mayor satisfacción con la relación de pareja. Moral (2008b) exploró la estructura factorial de los siete ítems de la RAS que mostraron consistencia interna buena ( $\alpha$ de Cronbach $=0,81$ ). El número de factores fue uno por el criterio de Kaiser. Tras la extracción del factor por Ejes Principales se explicó el 38,52\% de la varianza total. El ajuste a los datos del modelo unidimensional con siete indicadores fue adecuado por Máxima Verosimilitud: $\chi 2 / \mathrm{gl}=3.08$, $\mathrm{RMSEA}=0,07, \mathrm{GFI}=$ $0,92, \mathrm{AGFI}=0,98$ y CFI $=0,98$. En la presente muestra, la consistencia interna entre los siete ítems de la RAS fue excelente por el coeficiente alfa ordinal ( $\alpha$ ordinal $=0,93)$ y fue buena por el coeficiente alfa de Cronbach $(\alpha=0,89)$. Su distribución mostró asimetría negativa ( $\mathrm{s}=-0,87$, IC $95 \%:-1,04,-0,71)$ y apuntamiento ( $\mathrm{c}=$ 0,60, IC 95\%: $0,26,0,94)$, sin ajustarse a una curva 
normal (prueba de Kolmogorov-Smirnov-Lilliefors: $|\mathrm{D}|=0,102, \mathrm{p}<0,001)$.

\section{Procedimiento}

El estudio fue aprobado en sus aspectos éticos por el Comité de Doctorado, autoridad al respecto dentro de la Facultad de Psicología de la Universidad Autónoma de Nuevo León (UANL). Fue financiado por el Programa de Apoyo a la Investigación Científica y Tecnológica de la UANL, y no forma parte de ninguna tesis doctoral. Se solicitó el consentimiento expreso informado de los participantes en la primera hoja del cuestionario. En la hoja de consentimiento se garantizaba el anonimato de las respuestas y se informaba sobre la identidad del responsable del estudio, a quien se podía contactar por correo electrónico para cualquier cuestión suscitada por el estudio. No se solicitó ningún dato de identificación personal. De este modo, se respetaron las normas éticas de investigación de la American Psychological Association (2002).

\section{Análisis de datos}

En relación con el primer objetivo de estudiar las distribuciones de EEM y EEM14, se contrastó el ajuste a la normalidad por la prueba de Shapiro-Wilks, JarqueBera y Kolmogorov-Smirnov-Lilliefors. Se rechazó la hipótesis nula de normalidad con una probabilidad unilateral asintótica $\leq 0,05$. Las distribuciones se describieron por la media (M), desviación estándar (DE), cuantiles, coeficientes de asimetría (s) y curtosis (c) de Fisher, así como por el coeficiente de variación (cv) con un intervalo de confianza del 95\% desde el cálculo exacto de Verrill (2003).

En relación con el segundo objetivo de estudiar los correlatos de EEM y EEM14 con las variables sociodemográficas y de religiosidad, se emplearon pruebas no paramétricas debido a la falta de normalidad en la distribución de todas las variables numéricas. Se rechazó la hipótesis nula con una probabilidad bilateral $\leq 0,05$. Para estudiar la relación con las variables cualitativas se emplearon comparaciones de tendencia central. En el caso de dos grupos independientes (estado civil), las comparaciones se realizaron por la prueba u de Mann-Whitney-Wilcoxon. El tamaño del efecto se estimó por la correlación poliserial (rPs). Valores de rPs menores que 0,10 se interpretaron como un tamaño de efecto trivial; de 0,10 a 0,29 pequeño; de 0,30 a 0,49 moderado; de 0,50 a 0,69 grande; y mayor o igual que 0,70 muy grande (Olsson et al., 1982). Las comparaciones de tendencia central de cuatro grupos (afiliación religiosa) y ocho grupos (ocupación o actividad laboral) se ejecutaron por la prueba de Kruskal-Wallis. En caso de diferencia significativa, las comparaciones a posteriori se realizaron por la prueba de Dunn-Bonferroni. El tamaño de efecto se estimó por el coeficiente eta al cuadrado ( 2 2). Unos valores de $\eta 2$ menores que 0,01 se interpretaron como tamaño de efecto trivial; de 0,01 a 0,059 pequeño; de 0,06 a 0,139 mediano; y mayor o igual que 0,14 grande (Ellis, 2010). La relación con las variables numéricas y ordinales se estimó por el coeficiente de correlación de rangos ordenados de Spearman (rs). Unos valores de rs menores que 0,10 se interpretaron como una magnitud de asociación trivial; de 0,10 a 0,29 baja; de 0,30 a 0,49 moderada; de 0,50 a 0,69 alta; de 0,70 a 0,89 muy alta; $y$ mayor o igual que 0,90 unitaria (Ellis, 2010).

En relación con el tercer objetivo de estudiar la significación de los correlatos de EEM y EEM14 con las variables sociodemográficas y de religiosidad una vez controlado el efecto de la satisfacción con la relación (RAs), se empleó el coeficiente de correlación parcial de rangos ordenados de Spearman. Se contrastó la significación de la correlación parcial con una prueba t de Sudent con n - 2 grados de libertad (Sheskin, 2011). En el caso del estado civil se aplicó la fórmula de correlación parcial $\left(\mathrm{rxy} / \mathrm{z}=\left(\mathrm{rxy}-\left[\mathrm{rxz}^{\star} \mathrm{ryz}\right]\right) /\right.$ $\left.\left([1-\mathrm{rxz} 2]^{*}[1-\mathrm{ryz} 2]\right) 1 / 2\right)$, pero estimando las correlaciones del estado civil con la RAS, EEM y EEM14 por el coeficiente poliserial, y las correlaciones de la RAs con еEм y еEм14 por la correlación de Spearman. Se rechazó la hipótesis nula con una probabilidad bilateral $\leq 0,05$. Los cálculos se realizaron con spss versión 22 y el menú $\mathrm{R}$ versión 2,4 para SPSs22.

\section{Resultados}

\section{Descripción de la distribución de EEM Y EEM14}

La distribución de las puntuaciones en EEM (rango discreto de 0 a 18) fue asimétrica positiva $(s=0,31$, $E E=0,09)$ y platicúrtica $(c=-0,92, E E=0,17)$, y no se ajustó a una curva normal. La distribución mostró sesgo hacia puntuaciones por debajo de la media, por lo cual la media $(M=5,89$, IC $95 \%: 5,62,6,17)$ fue mayor que la mediana $(M d n=5)$. El cociente entre la desviación estándar y la media fue mayor que 0,33 ( $\mathrm{CV}$ $=0,68$, IC 95\%: 0,64, 0,73) (véanse tabla 1 y figura 1 ). 
La distribución de las puntuaciones en EEM14 (rango continuo de 1 a 7$)$ fue mesocúrtica $(C=-0,22$, $E E=0,17)$, y el cociente entre la desviación estándar y la media fue menor que 0,33 ( $C V=0,24$, IC $95 \%: 0,23$, $0,26)$. No obstante, la distribución mostró ligera asimétrica negativa $(s=-0,29, E E=0,09)$ y no se ajustó a una curva normal. El sesgo se presentó hacia puntuaciones por encima de la media, por lo cual la mediana $(M d n=4,50)$ fue mayor que la media $(M=4,43$, IC 95\%: $4,35,4,50)$ (véanse tabla 1 y figura 1). Los valores de tendencia central en EEM14 $(M=4,43$ y $M d n=$ $4,5)$ quedaron en el intervalo $[4,43,5,29]$ que corresponde a la quinta opción de respuesta de los ítems, la cual refleja que se está ligeramente de acuerdo con las afirmaciones de engrandecimiento marital.

\section{Relación con variables sociodemográficas}

\section{Estado civil}

La tendencia central de los casados en EEM y EEM14 fue significativamente mayor que la de las personas en unión libre, siendo el tamaño de efecto de la variable sociodemográfica pequeño, $r_{P S}<0,30$ (tabla 2).
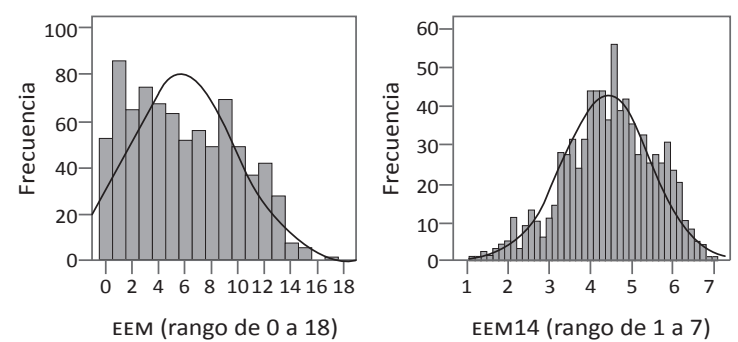

Figura 1. Histogramas de las distribuciones de EEM y EEM14. Elaboración propia.

Tabla 2

Comparación de tendencia central entre personas casadas y en unión libre

\begin{tabular}{|c|c|c|c|c|c|c|}
\hline \multirow[b]{2}{*}{$\begin{array}{l}\text { Escalas } \\
\text { (x) }\end{array}$} & \multicolumn{2}{|c|}{ Rango medio } & \multicolumn{2}{|c|}{ Mann-Whitney } & \multirow[b]{2}{*}{$r_{P S}[$ IC 95\%] } & \multirow[b]{2}{*}{$r(x y / z)$} \\
\hline & $\begin{array}{c}\text { Casados } \\
(n=727)\end{array}$ & $\begin{array}{c}\text { Unión libre } \\
\quad(n=80)\end{array}$ & $Z$ & $p$ & & \\
\hline EEM & 411,567 & 335,238 & $-2,788$ & 0,005 & $\begin{array}{c}-0,166^{* *} \\
{[-0,284,-0,048]}\end{array}$ & $-0,056^{n s}$ \\
\hline EEM14 & 412,244 & 329,081 & $-3,029$ & 0,002 & $\begin{array}{c}-0,180^{* * *} \\
{[-0,292,-0,068]}\end{array}$ & $-0,074^{*}$ \\
\hline
\end{tabular}

Tabla 1

Estadísticos descriptivos y pruebas de contraste de la normalidad

\begin{tabular}{ccc}
\hline Estadísticos & EEM $($ de 0 a 18) & EEM 14 (de 1 a 7) \\
\hline$M[$ IC 95\%] & $5,89[5,62,6,17]$ & $4,43[4,35,4,50]$ \\
$D E$ & 3,99 & 1,08 \\
$C V$ [IC 95\%] & $0,68[0,64,0,73]$ & $0,24[0,23,0,26]$ \\
$S[$ IC 95\%] & $0,31[0,14,0,48]$ & $-0,29[-0,46,-0,12]$ \\
$C$ IC 95\%] & $-0,92[-1,26,-0,58]$ & $-0,22[-0,56,0,12]$ \\
$P 10$ & 1 & 3,06 \\
$P 20$ & 2 & 3,50 \\
$P 25$ & 2 & 3,71 \\
$P 30$ & 3 & 3,93 \\
$P 40$ & 4 & 4,21 \\
$P 50$ & 5 & 4,50 \\
$P 60$ & 7 & 4,71 \\
$P 70$ & 9 & 5 \\
$P 75$ & 9 & 5,21 \\
$P 80$ & 10 & 5,43 \\
$P 90$ & 12 & 5,86 \\
$S W$ & $0,95^{* * *}$ & $0,99^{* * *}$ \\
$J B$ & $41,39^{* * *}$ & $12,94^{* *}$ \\
$K S L$ & $0,11^{* * *}$ & $0,03^{*}$ \\
\hline & &
\end{tabular}

Nota. $N=807 . M=$ Media. IC $=$ Intervalo de confianza. $D E=$ Desviación estándar. $\mathrm{CV}=$ Coeficiente de variación. $s=$ Asimetría o sesgo. $C=$ Curtosis. $P I=$ Percentil de orden i. $S W=$ Prueba de Shapiro-Wilks, $J B=$ Prueba de Jarque-Bera y $K S L=$ prueba de Kolmogorov-Smirnov-Lilliefors. Significación: $\mathrm{ns}=p>0,05,{ }^{*} p \leq 0,05$, ${ }^{* *} p \leq 0,01,{ }^{* * *} p \leq 0,001$. EEM $=$ Suma de los 18 ítems con puntuación dicotómica $(0,1) \cdot \operatorname{EEM} 14=(\mathrm{I} 1+[7-\mathrm{I} 2]+\mathrm{I} 3+\mathrm{I} 4+\mathrm{I} 5+\mathrm{I} 6+\mathrm{I} 8$ $+\mathrm{I} 9+\mathrm{I} 10+\mathrm{I} 12+\mathrm{I} 14+\mathrm{I} 15+\mathrm{I} 16+\mathrm{I} 18) / 14$. Elaboración propia.

Notas. $N=807 . r_{P S}=$ correlación poliserial. Estado civil $(\mathrm{y}): 1=$ Casado, $2=$ Unión libre. $\mathrm{z}=$ satisfacción con la relación $(\mathrm{RAS}) \cdot r_{P S}=-0,195$ entre RAS y estado civil. Elaboración propia. 


\section{Afiliación religiosa}

La tendencia central de los cuatro grupos de afiliación religiosa fue estadísticamente equivalente en EEM14 (K-W: $\chi^{2}=6.93, p=0,074$ ), pero hubo diferencia significativa en EЕM (K-W: $\left.\chi^{2}=10,40, p=0,015\right)$. El tamaño del efecto de la afiliación religiosa sobre EEM fue pequeño $\left(\eta^{2}=.013\right)$. Al hacer las comparaciones a posteriori, la tendencia central de las personas sin religión fue significativamente menor que la de las personas de otras religiones distintas a las cristianas ( $D=198,51$, IC $95 \%: 26,71,370,31, p<0,05)$ y que la de los cristianos no católicos ( $D=99,05$, IC 95\%: 0,77, $197,33, p<0,05$ ) (véase tabla 3 ).

\section{Variables sociodemográficas numéricas y ordinales}

La edad y los ingresos económicos fueron independientes de EEM y EEM14. El tiempo de relación fue independiente de EEM, pero tuvo una correlación baja con EEM14. La escolaridad, el número de hijos con la pareja actual y el número de parejas tuvieron correlaciones triviales con EEM y bajas con EEM14. Las dos variables de religiosidad y el número total de hijos tuvieron correlaciones bajas con la EEM y la EEM 14. En valores absolutos las correlaciones variaron de 0,001 a 0,21 con una media de 0,11 , siendo ocho de las 18 correlaciones $(44,4 \%)$ triviales (tabla 4 ).

Tabla 3

Comparaciones por pares en EEM14 por la prueba de Dunn-Bonferroni

\begin{tabular}{lcccc}
\hline \multicolumn{1}{c}{ Afiliación religiosa } & $\boldsymbol{n}$ & $\boldsymbol{R M}$ & Diferencia & $\boldsymbol{D}$ [IC 95\%] \\
\hline $\mathrm{G}_{1}=$ CRISTIANA CATÓLICA & 662 & 402,46 & $\mathrm{G}_{1}-\mathrm{G}_{2}$ & $-37,930[-105,517,29,657]$ \\
$\mathrm{G}_{2}=$ CRISTIANA NO CATÓLICA & 76 & 440,39 & $\mathrm{G}_{1}-\mathrm{G}_{3}$ & $-137,390[-293,677,18,897]$ \\
$\mathrm{G}_{3}$ = OTRA & 13 & 539,85 & $\mathrm{G}_{1}-\mathrm{G}_{4}$ & $61,120[-16,543,138,783]$ \\
$\mathrm{G}_{4}=$ NINGUNA & 56 & 341,34 & $\mathrm{G}_{2}-\mathrm{G}_{3}$ & $-99,460[-266,950,68,030]$ \\
& & & $\mathrm{G}_{2}-\mathrm{G}_{4}$ & $99,050^{*}[0,772,197,328]$ \\
& & & $\mathrm{G}_{3}-\mathrm{G}_{4}$ & $198,510^{*}[26,707,370,313]$ \\
\hline
\end{tabular}

Notas. $N=807 .{ }^{*} p<0,05 . R M=$ Rango medio. $D=$ Diferencia. Elaboración propia.

Tabla 4

Correlaciones bivariadas de las variables sociodemográficas y de religiosidad CON EEM, EEM14 y correlaciones parciales tras eliminar el efecto de RAS

\begin{tabular}{|c|c|c|c|c|c|}
\hline \multirow{2}{*}{$\begin{array}{c}\mathrm{x}=\text { Variables } \\
\text { sociodemográficas }(\mathrm{x})\end{array}$} & \multirow{2}{*}{$r_{s(\mathrm{xz})}$} & \multicolumn{2}{|c|}{$\mathrm{y}_{1}=\mathrm{EEM}$} & \multicolumn{2}{|c|}{$\mathrm{y}_{2}=\mathrm{EEM} 14$} \\
\hline & & $r_{s(x y 1)}$ & $r_{s(\mathrm{xy} 1 / \mathrm{z})}$ & $r_{s(\mathrm{xy} 2)}$ & $r_{s(\mathrm{xy} / \mathrm{z})}$ \\
\hline Edad & $-0,052 \mathrm{~ns}$ & $-0,001^{\mathrm{ns}}$ & $0,039^{\text {ns }}$ & $-0,070^{\mathrm{ns}}$ & $-0,048^{\mathrm{ns}}$ \\
\hline Escolaridad & $0,093^{* *}$ & $0,074^{*}$ & $0,022^{\mathrm{ns}}$ & $0,114^{* * *}$ & $0,071^{*}$ \\
\hline Ingresos económicos & $0,058^{\text {ns }}$ & $0,057^{\mathrm{ns}}$ & $0,027^{\mathrm{ns}}$ & $0,041^{\mathrm{ns}}$ & $0,005^{\mathrm{ns}}$ \\
\hline $\mathrm{N} .^{\circ}$ de hijos con la pareja & $-0,122^{\star * *}$ & $-0,082^{*}$ & $-0,010^{\mathrm{ns}}$ & $-0,133^{* * *}$ & $-0,073^{*}$ \\
\hline Número total de hijos & $-0,152^{* * *}$ & $-0,104^{* * *}$ & $-0,014^{\mathrm{ns}}$ & $-0,154^{* * *}$ & $-0,075^{*}$ \\
\hline N. ${ }^{\circ}$ de parejas previas & $-0,064^{\mathrm{ns}}$ & $-0,093^{\star *}$ & $-0,068^{\mathrm{ns}}$ & $-0,116^{* * *}$ & $-0,098^{* *}$ \\
\hline Tiempo de relación & $-0,075^{*}$ & $-0,039^{\text {ns }}$ & $0,009^{\text {ns }}$ & $-0,109^{\star *}$ & $-0,080^{*}$ \\
\hline Convicción religiosa & $0,162^{* * *}$ & $0,212^{* * *}$ & $0,145^{* * *}$ & $0,191^{* * *}$ & $0,116^{* * *}$ \\
\hline Práctica religiosa & $0,165^{* * *}$ & $0,198^{* * *}$ & $0,125^{* * *}$ & $0,186^{* * *}$ & $0,107^{*}$ \\
\hline
\end{tabular}

Nota. $\mathrm{z}=$ RAS. $r_{s(\mathrm{xy} 1 / \mathrm{z})}=$ Correlaciones entre EEM y las variables sociodemográficas y de religiosidad tras parcializar el efecto de RAS y $r_{s(\mathrm{xy} 2 / \mathrm{z})}$ = Correlaciones entre EEM14 y las variables sociodemográficas y de religiosidad tras parcializar el efecto de RAs, usando el coeficiente de correlación parcial de Spearman. Elaboración propia.

$r_{s(y 1 z)}=.610^{* * *} \mathrm{y} r_{s(y 2 z)}=.634^{* * *}$. 


\section{Actividad u ocupación laboral}

La tendencia central fue estadísticamente equivalente entre los ocho grupos de actividad u ocupación laboral tanto en EEM ( $\left.\mathrm{K}-\mathrm{W}: \chi^{2}=5,313, p=0,622\right)$ como en EEM (K-W: $\left.\chi^{2}=6,492, p=0,484\right)$. Consecuentemente, el tamaño del efecto estimado por el coeficiente eta fue trivial $\left(\eta^{2}<.01\right)$.

\section{Parcialización del efecto de la satisfacción con la relación}

Al parcializar el efecto de la satisfacción con la relación por correlación parcial, la relación entre estar casado o en unión libre con EEM dejó de ser significativa $\left(r_{(x y / z)}=-0,06, p>0,05\right)$. La relación con EEM14 permaneció significativa, aunque pasó a ser trivial $\left(r_{(x y / z)}=-0,07, p<0,05\right)$ (tabla 2$)$.

Si la comparación de medias de EEM entre los cuatro grupos de afiliación religiosa se hubiera realizado por el análisis de varianza, la diferencia entre las medias también sería significativa $(F[3,803]=3,47, p$ $=0,016$ ); no obstante, si se hubiera hecho por análisis de covarianza, introduciendo la satisfacción con la relación como covariable, entonces el efecto de la religión dejaría de ser significativo $(F[3,802]=2,05$, $p=0,105)$, cuando el efecto de la satisfacción con la relación sí sería significativo $(F[3,802]=362,40, p<$ $0,001)$.

Si se parcializa el efecto de la satisfacción con la relación, las correlaciones significativas de EEM con las variables sociodemográficas pasan a ser no significativas, pero se mantienen como significativas sus correlaciones con las dos variables de religiosidad. Tras esta parcialización, las correlaciones significativas de EEM14 con las variables sociodemográficas y de religiosidad siguen siendo significativas (tabla 4).

\section{Discusión}

En relación con el primer objetivo de describir la distribución de la versión original de la EEM con su rango discreto de 0 a 18 (O'Rourke y Cappeliez, 2001, 2002), se esperaba que las puntuaciones de la EEM presentaran una distribución con asimetría positiva y aplanamiento. Se confirmó la expectativa en esta muestra probabilística de parejas casadas y en unión libre de Monterrey. La distribución de EEM se desvió del modelo de una curva normal, presentó sesgo hacia los valores bajos, su perfil fue aplanado y la relación entre la desviación estándar y la media fue de aproximadamente dos tercios, lo cual se aleja del valor máximo de un tercio para series de valores positivos con distribución normal (Forkmana y Verrill, 2008). Aunque la variabilidad de la ЕЕм fue mayor que la correspondiente a una curva normal, esta no debe clasificarse como muy grande al ser el coeficiente de variación menor que uno, como sí podría ser el caso de una distribución exponencial o hipergeométrica (Forkmana y Verrill, 2008). El perfil del histograma fue bastante homogéneo entre los valores de 0 a 12. La amplitud y la variabilidad amplias indican que el fenómeno evaluado no es inusual, patológico o desadaptativo, pero no existe un nivel de expresividad claramente convencional (Cohen et al., 2012; Fischer y Milfont, 2010; Huang et al., 2009).

También, en relación con el primer objetivo de describir la distribución de la versión de 14 ítems con un rango continuo de 1 a 7 (Eem14; Moral, s. f. a), se esperaba que las puntuaciones de la EEM14 se ajustaran a un modelo de curva normal. No se confirmó la expectativa en esta muestra probabilística de parejas casadas y en unión libre de Monterrey. El perfil de la EEM14 fue mesocúrtico con un coeficiente de variación de aproximadamente un cuarto y unimodal, lo que la acerca a una curva normal, pero mostró ligero sesgo hacia las puntuaciones altas. Las tres pruebas estadísticas de contraste rechazaron la hipótesis nula de normalidad con una probabilidad menor que 0,05 , siendo la probabilidad menor que 0,01 en el caso de la prueba de Jarque-Bera, y menor que 0,001 en el caso de la prueba de Shapiro-Wilks. Con base en los estudios de simulación de la potencia de pruebas estadísticas para el contraste de la normalidad (Öztuna et al., 2006; Razali y Yap, 2011), las pruebas de Jarque-Bera y Shapiro-Wilks son las más potentes, pues alcanzan una potencia unitaria con tamaños de muestra $>500$, por lo cual la inferencia de desviación de la normalidad a causa de esta asimetría negativa es clara. No obstante, la desviación de la normalidad que presenta la EEM14 debe clasificarse como una desviación no extrema, ante la cual se pueden emplear pruebas de contraste paramétricas si se tiene una muestra grande (Rochon et al., 2012). Este perfil próximo a una curva normal con un nivel promedio claramente especificable se ajusta mejor al concepto de rasgo adaptativo que se pretende medir con la escala (O’Rourke y Cappeliez, 2002) que el perfil de la EEM. La normalidad o forma de campana en la distribución de las puntuaciones de una variable que evalúa un rasgo funcional viene determinada por las presiones y reglas ambientales de adaptación. Existe una 
intensidad expresiva del rasgo medido que optimiza la adaptación en un nicho ecológico, la cual se encuentra en la mayoría de los individuos de ese entorno; por el contrario, una expresión desviada por exceso o defecto de esa tendencia central (media, mediana o moda) suele resultar en desventajas, por lo que es presentada por una minoría de individuos. Sin embargo, cambios ambientales pueden provocar que una manifestación excesiva o defectuosa del rasgo resulte en mayores ventajas adaptativas, desplazando la tendencia central poblacional (Rushton, 2012).

Se observa un sesgo hacia las puntuaciones altas en la ЕEм14 dentro de la población estudiada, lo cual es congruente con la teoría de la reducción de la disonancia ante unos valores culturales de monogamia o elección de pareja de por vida (O’Rourke et al., 2011; Ubillos et al., 2001). Considerando que la media de años de vivir juntos se aproxima a 12 años, una idealización extrema o ciega consecuencia de un enamoramiento pasional no es esperable, pero sí una ligera o moderada idealización (Sánchez-Aragón, 2006). La media de la población se ubicó con una ligera conformidad y una calificación muy positiva de la pareja y de la relación. Precisamente esta tendencia a la ligera idealización se puede explicar desde la justificación de elección de pareja (Gagné y Lydon, 2004; Sánchez-Aragón, 2006); así mismo, desde el deseo de conservar una relación positiva y amorosa con dicha persona, esto es, desde la experiencia positiva de apoyo y seguridad (Fowers y Applegate, 1996).

Con base en los solapamientos de los intervalos, la media de la ЕEM (versión en español) obtenida en la presente muestra $(M=5,89$; IC $95 \%$ : $5,62,6,17)$ es estadísticamente equivalente $(p>0,05)$ a la reportada por O'Rourke y Cappeliez (2001) en una muestra de 532 adultos mayores canadienses anglófonos con la versión original de la EEM en inglés $(M=5,53$; IC 95\%: 5,11, 5,94); a la reportada por O'Rourke (2002) en el estudio de seguimiento de 198 adultos mayores canadienses anglófonos con la misma versión $(M=$ 5.46; IC 95\%: 4,83, 6,09); a la reportada por O'Rourke y Cappeliez (2003b) en una muestra de 61 adultos mayores canadienses francófonos con la versión de la EEM en francés ( $M=5,84$, IC $95 \%: 5,66,6,03)$; a la reportada por O’Rourke et al. (2012) en una muestra de 206 adultos mayores israelíes con la versión de la EEM en hebreo ( $M=5,48$, IC $95 \%: 4,85,6,11)$; así como a la reportada por Moral (2007) en una muestra de 200 adultos jóvenes mexicanos con la versión en español $(M=5,87 ;$ IC 95\%: 5,32, 6,42). Así mismo, la media de la EEM14 obtenida en la presente muestra $(M=4,43$, IC $95 \%: 4,35,4,50)$ es estadísticamente equivalente ( $p$ $>0,05)$ a la reportada por Moral (2007) en una muestra extraída de la misma población $(M=4,25$; IC $95 \%$ : $4,13,4,37)$, por lo cual el nivel de engrandecimiento parece bastante homogéneo entre países con diferencias culturales importantes.

En relación con el segundo objetivo de estudiar la relación de la EEM y la EEM14 con 12 variables sociodemográficas, se confirmó la expectativa de independencia o correlación baja. Debe señalarse que los promedios de la EEM y la EEM14 son estadísticamente equivalentes entre mujeres y hombres en esta misma muestra, como previamente se ha reportado en otro estudio sobre la invarianza de las escalas por sexo (Moral, s. f. b). Desde los presentes datos, la ocupación laboral, la edad y los ingresos económicos fueron independientes de la EEM y la EEM14. Además, el tiempo de relación con la pareja tuvo un efecto mínimo o trivial. Los siete correlatos sociodemográficos, con relaciones bajas o triviales, muestran que la idealización de la pareja es mayor cuanto menor es el número de hijos totales y con la pareja, menor es el número de parejas previas, mayor es el nivel de escolaridad y menor es el tiempo de relación con la pareja. Así mismo, evidencian que la idealización de la pareja es mayor en las personas casadas que en las que están en unión libre, y que es mayor en las personas adscritas a una religión cristiana no católica u otra religión distinta a la cristiana que en los cristianos católicos y las personas sin religión. Estas correlaciones y diferencias coinciden con las encontradas en los estudios de satisfacción con la relación (Wheeler et al., 2010), y duración de la relación (Margelisch et al., 2015), y son concordantes con el constructo (O'Rourke y Cappeliez, 2002).

Se señala que el tiempo de relación va atenuando el enamoramiento y la idealización de la pareja por efecto de habituación (Gagné y Lydon, 2004; Margelisch et al., 2015), lo cual puede explicar la correlación negativa. En comparación con aquellas personas que han tenido solo una pareja con la que se han casado, el hecho de no casarse con la pareja, haber tenido varias parejas previas (en unión libre o en matrimonio) e hijos fuera del matrimonio suele reflejar una imagen más crítica o realista de la pareja y de menor compromiso y entrega (Sánchez-Aragón, 2006); de ahí las correlaciones negativas con idealización de la pareja y la relación. Observar menor engrandecimiento a mayor número de hijos se puede atribuir al 
efecto de pérdida de intimidad y los conflictos que genera en la pareja una prole creciente (Hirschberger et al., 2009). La menor idealización entre las personas de menor escolaridad probablemente esté mediada por matrimonios más tempranos (más tiempo de casados), paternidad más temprana (más conflictos de pareja) y mayor número de hijos que son características de la población (INEGI, 2011a; Ubillos et al., 2001). La mayor idealización entre creyentes no católicos probablemente se deba a la mayor fe y fervor en su religión no mayoritaria dentro de la población mexicana, en la cual el culto católico es dominante, pero viene sufriendo una pérdida lenta de adeptos (INEGI, 2011b).

En relación con el tercer objetivo de comprobar la sustantividad de estos siete correlatos una vez parcializado el efecto de la satisfacción con la pareja, se observó que las únicas variables con relaciones significativas tanto en EEM como en EEM14 serían las dos variables de religiosidad, cuya magnitud de correlación se reduciría a la mitad. Concordante con los valores religiosos de monogamia y fidelidad (Ubillos et al., 2001), la idealización de la pareja es mayor cuanto mayor es la convicción religiosa y la asistencia a los servicios religiosos, siendo ligeramente mayor el efecto de la fe que el de la práctica religiosa. En cierto modo, la idealización se podría reinterpretar como una fe ciega en la pareja (Goddard et al., 2012). La idealización tiene lugar en un plano cognitivo y en una esfera personal, como la convicción religiosa. No corresponde tanto a una práctica social consensuada y reforzada en una escena pública como la práctica religiosa (Singleton, 2014). Los otros cinco correlatos sociodemográficos de EEM resultaron no significativos una vez parcializado el efecto de la satisfacción con la pareja, como previamente habían reportado con la versión inglesa (O’Rourke y Cappeliez, 2002) y francesa (O’Rourke y Cappeliez, 2003b), lo que indica que son correlaciones espurias explicadas por las diferencias sociodemográficas en satisfacción con la pareja. Los cuatro correlatos sociodemográficos de EEM14 permanecieron como significativos, aunque pasaron a ser triviales $(<0,10)$, lo que resulta en un efecto sustantivo mínimo que no requiere la elaboración de baremos diferenciales o de control al momento del diseño de un estudio o el análisis de los datos sobre engrandecimiento marital.

Puede llamar la atención un rango de edad tan amplio en la presente muestra (de 18 a 94 años). Si se define un valor atípico leve como aquel que rebasa al tercer cuartil $\left(Q_{3}\right)$ en una vez y media el valor del rango intercuartílico $\left(Q_{3}+\left[1,5^{*} R_{I Q}\right]=43+\left[1,5^{\star} 15\right]=\right.$
65,5; $\left.R_{\mathrm{IQ}}=\mathrm{Q}_{3}-\mathrm{Q}_{2}=43-28=15\right)$, habría dos casos $(71$ y 94 años). Si se define un valor atípico extremo como aquel que rebasa al tercer cuartil $\left(Q_{3}\right)$ en tres veces el valor del rango intercuartílico $\left(Q_{3}+\left[3^{*} R_{I Q}\right]=43+\right.$ $\left.\left[3^{\star} 15\right]=88\right)$, habría un caso, el cual corresponde a un adulto de la cuarta edad (94 años). Al no alterar los cálculos de tendencia central, variación y covarianza en EEM y EEM14, la inclusión de este caso extremo, tener todos sus datos completos, no definir ningún caso atípico en ninguna de las otras variables analizadas y considerando que los adultos mayores de 80 o más años es un grupo de edad que está presente en la ciudad, el participante de 94 años fue incluido en el estudio. Si se considera el percentil 95 de la distribución de la edad $\left(P_{95}=53\right)$, se podría definir un grupo de 763 participantes con 52 o menos años, y un grupo de 44 participantes con 53 o más años. Como en el resto de la muestra, la edad fue independiente de la puntuación EEM y EEM14 entre los 44 participantes con 53 o más años. Además, la media en la escala EEM y EEM14 fue estadísticamente equivalente entre estos dos grupos de edad, ya sea que el contraste se haga por la prueba t de Student o la u de Mann-Whitney, lo cual refuerza la conclusión de que los adultos mayores no constituyen un grupo especial en relación con el engrandecimiento marital.

Como limitación del presente estudio debe señalarse que su generalización solo es válida para la población de parejas casadas o en unión libre de la ciudad de Monterrey en México. En otras poblaciones urbanas de México, o de países económica y culturalmente afines, deben manejarse como hipótesis. Así mismo, debe especificarse que los presentes datos son de autorreporte y podrían diferir de datos obtenidos por medio de entrevistas abiertas.

\section{Conclusión}

En la población estudiada, la distribución de las puntuaciones en EEM mostró aplanamiento y sesgo hacia las puntuaciones bajas con clara desviación de la normalidad, por lo cual no puede considerarse como una variable paramétrica y el uso de cuantiles constituye la forma de baremación apropiada. Su distribución bastante homogénea refleja una característica sin nivel de expresividad claramente convencional, aunque no patológica, inusual o rara. La distribución de las puntuaciones en EEM14 fue unimodal, mesocúrtica y con ligero sesgo hacia las puntuaciones altas, por lo que mostró una ligera desviación de la normalidad, aunque tolerable con pruebas paramétricas siempre que el tamaño de muestra sea grande. Se podría 
baremar por cuantiles o por la media y la desviación estándar. Su distribución muestra una característica usual con un nivel específico de convencionalidad, lo que es congruente con el concepto de rasgo adaptativo que se pretende medir. En esta población la tendencia promedio es de ligera idealización de la pareja, lo cual es explicable desde los valores culturales de monogamia en relación con la teoría de la reducción de la disonancia, así como desde la experiencia de una relación positiva y de apoyo con la pareja íntima. Se confirma la independencia o baja relación de EEM y EEM14 con las variables sociodemográficas de edad, escolaridad, estado civil (casado o en unión libre), afiliación religiosa, ingresos económicos, número de hijos con la pareja y número total de hijos, número de parejas previas y tiempo de relación con la pareja; así mismo, se confirma la expectativa de relaciones espurias (con EEM) o triviales (con EEM14), una vez eliminado el efecto de la satisfacción con la relación. No obstante, tanto EEM como EEM14 presentan una correlación baja, pero sustantiva, con la religiosidad. Se observa que cuanto mayor es la creencia religiosa y la práctica de la religión, mayor es la tendencia a idealizar a la pareja.

Debe señalarse que la tendencia central de los hombres fue estadísticamente equivalente a la de las mujeres, tanto en EEM como en EEM14, al ser contrastada por la prueba u de Mann-Whitney (Moral, s. f. b), por lo cual los baremos reportados se pueden emplear en ambos sexos, lo que da gran facilidad de uso a la escala. Se sugiere el uso de la EEM14 en los estudios de pareja en México, ya sea como una variable predictora o de control. A efectos prácticos se puede considerar como paramétrica. Si se toman la media y la desviación como normas interpretativas, una puntuación mayor o igual que 5,51 muestra una tendencia por encima de lo común a idealizar a la pareja, y por el contrario una puntuación menor o igual a 3,34 muestra una tendencia por debajo de lo común dentro de la población de parejas casadas y en unión libre de Monterrey. Además, se sugiere el estudio de la validez criterial de la escala, estimando el sesgo a la idealización de la pareja y la relación con una prueba proyectiva o una entrevista de preguntas abiertas (criterios de comparación).

\section{Referencias}

Ahmadi, K., Azad-Marzabadi, E. y Nabipoor, A. S. M. (2008). The influence of religiosity on marital satisfaction. Journal of Social Sciences, 4, 103-110. doi: 10.3844/jssp.2008.103.110
American Psychological Association. (2002). Ethical principles of psychologists and code of conduct. American Psychologist, 57, 1060-1073. doi: 10.1037/ 0003-066X.57.12.1060

Bodenmann, G., Ledermann, T. y Bradbury, T. N. (2007). Stress, sex, and satisfaction in marriage. Personal Relationships, 14, 551-569. doi:10.1111/j.14756811.2007.00171.x

Boyes, A. D. y Fletcher, G. J. O. (2007). Metaperceptions of bias in intimate relationships. Journal of Personality and Social Psychology, 92, 286-306. doi: 10.1037/00223514.92.2.286

Britt, S., Grable, J. E., Goff, B. S. N. y White, M. (2008). The influence of perceived spending behaviors on relationship satisfaction. Financial Counseling and Planning, 19(1), 31-43.

Carlson, R. G., Barden, S. M., Daire, A. P. y Greene, J. (2014). Influence of relationship education on relationship satisfaction for low-income couples. Journal of Counseling \& Development, 92, 418-427. doi: 10.1002/j.1556-6676.2014.00168.x

Cohen, R. J., Swerdlik, M. y Sturman, E. D. (2012). Psychological testing and assessment: An introduction to tests and measurement (8. ${ }^{\mathrm{a}}$ ed.). Nueva York: McGraw-Hill.

Dellner, D. K. (2008). Pornography use, relationship functioning and sexual satisfaction: The mediating role of differentiation in committed relationships (Tesis doctoral). Recuperado de la base de datos de ProQuest Dissertations \& Theses (UMI N. ${ }^{\circ} 3335233$ ).

Díaz-Loving, R. y Rivera-Aragón, S. (2010) (Eds.). Antología psicosocial de la pareja. Ciudad de México: unAm y Editorial Miguel Ángel Porrúa.

Edmonds, V. H. (1967). Marital conventionalization: Definition and measurement. Journal of Marriage and the Family, 29, 681-688. doi: 10.2307/349220

Ellis, P. D. (2010). The essential guide to effect size. Cambridge, uk: Cambridge University Press.

Fanni-Asl, A., Narimani, M., Rajabi, S. y Siahpoosh, H. (2009). The study of religious behavior and faith in relation with student's marital satisfaction. Research Journal of Biological Science, 4(1), 42-47.

Fischer, R. y Milfont, T. L. (2010). Standardization in psychological research. International Journal of Psychological Research, 3(1), 88-96.

Forkmana, J. y Verrill, S. (2008). The distribution of McKay's approximation for the coefficient of variation. Statistics and Probability Letters, 78, 10-14. doi: 10.1016/j. spl.2007.04.018 
Fowers, B. J. y Applegate, B. (1996). Marital satisfaction and conventionalization examined dyadically. Current Psychology, 15, 197-214. doi: 10.1007/BF02686877

Gagné, F. y Lydon, J. (2004). Bias and accuracy in close relationships: An integrative review. Personality and Social Psychology Review, 8, 322-338. doi: 10.1207/ s15327957pspr0804_1

Glenwright, B. J. y Fowler, D. M. (2013). Implications of egalitarianism and religiosity on relationship satisfaction. Interpersona, 7, 215-226. doi:10.5964/ijpr.v7i2.137

Goddard, H. W., Marshall, J. P., Olson, J. R. y Dennis, S. A. (2012). Character strengths and religiosity as predictors of marital satisfaction in a sample of highly religious and divorce-prone couples. Journal of Couple and Relationship Therapy: Innovations in Clinical and Educational Interventions, 11(1), 2-15. doi:10.1080/15 332691.2011 .613308

Hendrick, S. S. (1988). A generic measure of relationship satisfaction. Journal of Marriage and the Family, 50, 9398. doi: $10.2307 / 352430$

Hirschberger, G., Srivastava, S., Marsh, P., Cowan, C. y Cowan, P. (2009). Attachment, marital satisfaction, and divorce during the first fifteen years of parenthood. Personal Relationships, 16, 401-420. doi: 10.1111/j.1475-6811.2009.01230.x

Huang, Y., Kotov, R., De Girolamo, G., Preti, A., Angermeyer, M., Benjet, C., ..., Kessler, R. C. (2009). DsM-IV personality disorders in the wHo World Mental Health Surveys. The British Journal of Psychiatry, 195, 46-53. doi:10.1192/bjp.bp.108.058552

Instituto Nacional de Estadística, Geografía e Informática (2011a). Panorama sociodemográfico de Nuevo León. Ciudad de México: INEgI.

Instituto Nacional de Estadística y Geografía (2011b). Panorama de las religiones en México 2010. Ciudad de México: INEGI y Secretaría de Gobernación.

Jackson, J. B., Miller, R. B., Oka, M. y Henry, R. G. (2014). Gender differences in marital satisfaction: A meta-analysis. Journal of Marriage and Family, 76(1), 105-129. doi:10.1111/jomf.12077

Jose, O. y Alfons, V. (2007). Do demographics affect marital satisfaction? Journal of Sex and Marital Therapy, 33, 73-85. doi:10.1080/00926230600998573

Margelisch, K., Schneewind, K. A., Violette, J. y Perrig-Chiello, P. (2015). Marital stability, satisfaction and well-being in old age: variability and continuity in long-term continuously married older persons. Aging and Mental Health, 10, 1-10. doi:10.1080/13607863.2015.1102197
Moral, J. (2007). Control de la deseabilidad social ante la evaluación del ajuste diádico y la satisfacción marital. Psicología y Ciencia Social, 9(2), 5-12.

Moral, J. (2008a). Predicción del ajuste diádico en una muestra nuevoleonesa. Revista Interamericana de Psicología, 42(2), 1-10.

Moral, J. (2008b). Validación de la Escala de Valoración de la Relación en población mexicana. Revista Electrónica de Metodología Aplicada, 13(1), 1-12. Recuperado de http://www.psico.uniovi.es/REMA/v13n1/vol13n1a1. pdf

Moral, J. (2015). Diferencias en satisfacción con la relación entre variables demográficas y religiosidad. Revista Salud \& Sociedad, 6(1), 122-137.

Moral, J. (s. f. b). Consistencia interna y validez estructural y convergente de la Escala de Engrandecimiento Marital en una muestra probabilística de Monterrey, México. Revista Iberoamericana de Diagnóstico y Evaluación Psicológica, 42.

Moral, J. (s. f. b). Invarianza factorial de la Escala de Engrandecimiento Marital por sexo. Informes Psicológi$\cos , 16(2)$.

Olsson, U., Drasgow, F. y Dorans, N. J. (1982).The polyserial correlation coefficient. Psychometrika, 47, 337-347. doi:10.1007/BF02294164

O'Rourke, N. (2001). Development and validation of a couples measure of biased responding: The Marital $\mathrm{Ag}$ grandizement Scale (Tesis doctoral, University of Ottawa). Recuperado de http://www.ruor.uottawa.ca/ bitstream/10393/9347/1/NQ66177.PDF

O'Rourke, N. (2002). Predictors of change in marital aggrandizement among older adult. Journal of the Aging Family System, 1, 75-85.

O'Rourke, N. y Cappeliez, P. (2001). Marital satisfaction and marital aggrandizement among older adults: Analysis of gender invariance. Measurement and Evaluation in Counseling and Development, 34, 66-79.

O'Rourke, N. y Cappeliez, P. (2002). Development and validation of a couples measure of biased responding: The Marital Aggrandizement Scale. Journal of Personality Assessment, 78, 301-320. doi: 10.1207/ S15327752JPA7802_06

O'Rourke, N. y Cappeliez, P. (2003a). Intra-couple variability in marital aggrandizement: Idealization and satisfaction within enduring relationships. Current Research in Social Psychology, 8(15), 206-225.

O'Rourke, N. y Cappeliez, P. (2003b). Validation d'une mesure de réponses biaisées à propos de la relation 
conjugale: L'Échelle d'embellissement conjugal. Revue canadienne des sciences du comportement, 35, 45-49. doi: $10.1037 / \mathrm{h} 0087185$

O’Rourke, N., Claxton, A., Kupferschmidt, A. L., Smith, J. Z. y Beattie, B. L. (2011). Marital idealization as an enduring buffer to distress among spouses of persons with Alzheimer disease. Journal of Social and Personal Relationships, 28(1), 117-133. doi: 10.1177/ 0265407510386135

O’Rourke, N., Gomes, Y. y Davidov, E. (2012). Marital idealization, caregiver burden, and life satisfaction among spouses of persons with Alzheimer disease. Gerontology and Geriatrics, 29, 91-110.

O’Rourke, N., Haverkamp, B. E., Rae, S., Toukko, H., Hayden, S. y Beattie, B. (1996). Response biases as a confound to expressed burden among spousal caregivers of suspected dementia patients. Psychology and Aging, 11, 377-380. doi: 10.1037/0882-7974.11.2.377

O'Rourke, N., Polchenko, N., Bachner, Y. G. y Carmel, S. (2012). Translation and validation of a Hebrew language version of the Marital Aggrandizement Scale. Ponencia presentada en The Annual Meeting of the American Psychological Association, Orlando, Florida, Estados Unidos, agosto de 2012. Recuperado de http://www.researchgate.net/publication/252931460

O’Rourke, N. y Tuokko, H. (2000). The psychological and physical cost of caregiving: The Canadian Study of Health and Aging. Journal of Applied Gerontology, 19, 389-404. doi: 10.1177/073346480001900402

O’Rourke, N. y Wenaus, C. A. (2010). Marital aggrandizement as a mediator of burden among spouses of suspected dementia patients. Canadian Journal on Aging, 17, 384-400. doi: 10.1017/S0714980800012666

Öztuna, D., Elhan, A. H. y Tüccar, E. (2006). Investigation of four different normality tests in terms of type 1 error rate and power under different distributions. Turkish Journal of Medical Sciences, 36(3), 171-176.

Paulhus, D. L. (1998). Manual for balanced inventory of desirable responding: Version 7 (BIDR-7). Toronto: Multi-Health Systems.

Razali, N. M. y Yap, B. W. (2011). Power comparisons of Shapiro-Wilk, Kolmogorov-Smirnov, Lilliefors, and Anderson-Darling test. Journal of Statistical Modeling and Analytics, 2(1) 21-33.
Rochon, J., Gondan, M. y Kieser, M. (2012). To test or not to test: Preliminary assessment of normality when comparing two independent samples. BMC Medical Research Methodology, 12, 81. doi: 10.1186/1471-228812-81.

Rushton, P. (2012). Race, evolution, and behavior: A life history perspective. New Brunswick: Transaction Publishers.

Sampieri, R., Fernández, C. y Baptista, P. (2010). Metodología de la investigación. Ciudad de México: McGraw-Hill.

Sánchez-Aragón, R. (2006). Pasión romántica: más allá de la intuición, una ciencia del amor. Ciudad de México: Miguel Ángel Porrúa.

Sheskin, D. J. (2011). Handbook of parametric and nonparametric statistical procedure (5. ${ }^{\mathrm{a}}$ ed.). Boca Ratón: CRC Press.

Singleton, A. (2014). Religion, culture and society: A global approach. Los Angeles: Sage.

Spanier, G. B. (1976). Measuring dyadic adjustment: New scales for assessing the quality of marriage and similar dyads. Journal of Marriage and the Family, 38, 15-28. doi: $10.2307 / 350547$

Swami, V., Stieger, S., Haubner, T., Voracek, M. y Furnham, A. (2009). Evaluating the physical attractiveness of oneself and one's romantic partner: Individual and relationship correlates of the love-is-blind bias. Journal of Individual Differences, 30(1), 35-43. doi: 10.1177/0265407510385492

Ubillos, S., Zubieta, E., Páez, D., Deschamps, J. C., Ezeiza, A. y Vera, A. (2001). Amor, cultura y sexo. Revista Electrónica de Motivación y Emoción, 4, 8-9.

Verrill, S. (2003). Confidence bounds for normal and lognormal distribution coefficients of variation. Madison: United States Department of Agriculture. Recuperado de http://www1.fpl.fs.fed.us/fplrp609.pdf

Wendorf, C. A., Lucas, T., Imamoglu, E. O., Weisfeld, C. C. y Weisfeld, G. E. (2011). Marital satisfaction across three cultures: Does the number of children have an impact after accounting for other marital demographics? Journal of Cross-Cultural Psychology, 42(3), 340-354. doi: $10.1177 / 0022022110362637$

Wheeler,L.A., Updegraff, K.A.yThayer, S.M.(2010).Conflict resolution in Mexican-origin couples: Culture, gender, and marital quality. Journal of Marriage and the Family, 72, 991-1005. doi: 10.1111/j.1741-3737.2010.00744.x 